\title{
Gehen Sie nicht über Los! Eine Erwiderung auf Hubertus Buchstein
}

\author{
Roland Lhotta
}

\section{Los und Zufall in der parlamentarischen Demokratie: Das "große Los"?}

Lostrommel oder Wahlurne - das ist eine Alternative, die zum Widerspruch herausfordern muss. Dessen ist sich Hubertus Buchstein bei seinem mutigen Plädoyer für den „Zufall mit Absicht" durchaus bewusst, denn selbst die aus seiner Sicht niederschwelligste Variante des Einsatzes von Losverfahren - als ultima ratio, wenn gar nichts mehr geht, wie etwa beim „Justiz-Lotto“1 im Kontext des Münchener NSU-Verfahrens - lässt angesichts der desaströsen Eindrücke, die hiervon zurückgeblieben sind $^{2}$, erhebliche Zweifel aufkommen. Die von ihm präsentierten Überlegungen zur aleatorischen Demokratietheorie sind insofern eine gewisse Provokation, und diese Provokation ist - trotz seines Plädoyers für den Zufall - natürlich kein Zufall. Gut so! Denn Provokationen fordern zur Auseinandersetzung heraus und es ist zunächst einmal ein Hauptanliegen der Verfechter einer aleatorischen Demokratie, solche Auseinandersetzungen - besser noch: Deliberationen - in Gang zu bringen.

Dazu hat mein Vorredner die besten Voraussetzungen geschaffen, indem er nicht nur im Rahmen seines Vortrags, sondern vor allem auch in Publikationen der letzten Jahre eine ganze Reihe von Argumenten präsentiert hat, mit denen man sich befassen sollte. ${ }^{3}$ Dies ist zugegebenermaßen nicht ganz einfach, denn die aleatorische Demokratietheorie ist ein Thema, das nicht gleich Affinitäten zu wecken vermag. Auch wenn es sich um ein seit der Antike bekanntes Verfahren zur Besetzung von Ämtern und zur Herbeiführung von Entscheidungen handelt ${ }^{4}$ - der auf Rationalität, Machbarkeit, Beherrschbarkeit und Kontrolle gegründete moderne Staat und die moderne Demokratie einerseits sowie die Aleatorik andererseits scheinen weitgehend inkommensurabel. Mehr noch: Argumente für die Aleatorik führen oft zu Irritationen, zu Befremden und zu Abwehrreflexen ${ }^{5}$, weil es hier um nicht

1 Reinhard Müller, Justiz-Lotto, in: FAZ.NET vom 19. April 2013, http://www.faz.net/-hqo-78j3m (Abruf am18. Mai 2013).

2 Vgl. dazu Frank Lübberding, Das große Los, in: FAZ.NET vom 1. Mai 2013, http://www.faz. net/-gqz-78t63; Kurt Kister, Eine Liste wie eine Farce, in: Süddeutsche.de vom 29. April 2013, http://www.sueddeutsche.de/politik/akkreditierung-beim-nsu-prozess-eine-liste-wie-eine-farce-1.1661810; Annette Ramelsberger, 50 aus 324 - Glücksspiel am Gericht, in: Süddeutsche.de vom 29. April 2013, http://www.sueddeutsche.de/politik/presseplaetze-fuer-nsu-prozess-ausgluecksspiel-am-gericht-1.1661748 (jeweils Abruf am 19. Mai 2013).

3 Vgl. hierzu vor allem Hubertus Buchstein, Bausteine zu einer aleatorischen Demokratietheorie, in: Leviathan, 37. Jg. (2009), H. 3, S. 327 - 352; ders., Reviving Randomness for Political Rationality: Elements of a Theory of Aleatory Democracy, in: Constellations, 17. Jg. (2010), H. 3, S. 435 - 454; ders., Wählen, Losen und politische Gerechtigkeit - Plädoyer für einen ,demokratischdeliberativen pouvoir neutre', in: ZPol, 22. Jg. (2012), H. 3, S. 397 - 407; ders.; Elective and Aleatory Parliamentarism (bislang Manuskript), in: Kari Palonen / José Maria Rosales (Hrsg.), Parliamentarism and Democratic Theory. Historical and Contemporary Perspectives, 2014 (im Erscheinen).

4 Vgl. hierzu ders., Demokratie und Lotterie. Das Los als politisches Entscheidungsinstrument von der Antike bis zur EU, Frankfurt am Main 2009.

5 Es ist übrigens bemerkenswert, dass sich solche Reflexe auch schon in frühchristlichen Schriften wie der dem cyprianischen Umfeld zugeschriebenen Predigt „Adversus Aleatores“ finden - hier 
weniger als um einen Bruch mit modernen Standardvorstellungen von Politik und Demokratie geht ${ }^{6}$, zumindest aber um eine „größere Distanz zu den gewohnten Institutionen und Verfahren der nationalstaatlich organisierten Demokratien“7 sowie um „reformpolitisch weiterführende Vorschläge“ in Richtung einer „Transformation der Demokratie“8.

Diese Vorschläge wiederum haben einen beachtlichen Konkretionsgrad; sie zielen auf (experimentelle) institutionelle Reformen ${ }^{9}$ und haben einen stark praxisorientierten Impetus, der mitten ins Herz der tradierten Formen repräsentativer und das heißt natürlich vor allem: parlamentarischer, mithin von Parteien dominierter Demokratie zielt - selbstverständlich in guter und konstruktiver Absicht, denn es soll ja gerade die Legitimität parlamentarischer Demokratie durch den Einsatz des „Zufalls mit Absicht“ gestärkt werden. Und damit nicht genug: In Aussicht gestellt werden des weiteren Zugewinne an Rationalität, Deliberation, Gerechtigkeit, Gleichheit, Effizienz, Partizipation, Repräsentativität, Responsivität und Neutralität. ${ }^{10}$ Parbleu! Bei solchen Aussichten stellt sich die Alternative Lostrommel oder Wahlurne doch gleich in einem milderen Licht dar - wenn man nicht gar sofort beherzt zur Lostrommel greifen oder dem Präsidenten des Bundestages die Anschaffung einiger Bündel Strohhälmchen empfehlen wollte, um endlich einmal mehr Vertrauen in den Zufall zu demonstrieren. „Gehen Sie über Los"11 - die Empfehlung von Hubertus Buchstein scheint - gerade auch vor dem Hintergrund des beeindruckenden ideengeschichtlichen und verfahrenshistorischen Introitus seiner Ausführungen - fast unwiderstehlich. Aber zieht man wirklich das "große Los“, wenn man sich auf die (partielle und substitutive) Institutionalisierung des Zufallsprinzips im Rahmen einer repräsentativen Demokratie einlässt? Hier sind dann doch einige Zweifel angebracht!

\section{Von der Badstraße und vom Gefängnis, oder: Gehe nicht über Los!}

„Gehe über Los“ - vielen von Ihnen könnte das bekannt vorkommen. Diese Aufforderung entstammt einer Ereigniskarte aus dem bekannten Gesellschaftsspiel Monopoly - ein Spiel,

werden vor allem die Verderbtheit des Glücksspiels sowie die Hingabe an den Zufall in derben Worten gebrandmarkt und als Versündigung gegen den heiligen Geist und des Teufels hingestellt.

6 Dies hat insbesondere die anregende Studie von Bernard Manin, The Principles of Representative Government, Oxford 1997 gezeigt, in der er auf die ursprüngliche Bedeutung von Losverfahren in der (direkten) athenischen Demokratie verweist und zeigt, wie es geschichtlich zu einer völligen Identifikation von Wahlen mit der repräsentativen Demokratie kam: „The fact that representative governments have never used lot to assign political power shows that the difference between the representative system and ,direct' systems has to do with the method of selection rather than with the limited number of those selected. What makes a system respresentative is not the fact that a few govern in the place of the people, but that they are selected by election only" (S. 41).

7 Hubertus Buchstein / Michael Hein, Zufall mit Absicht. Das Losverfahren als Instrument einer reformierten Europäischen Union, in: Hauke Brunkhorst (Hrsg.), Demokratie in der Weltgesellschaft (Soziale Welt, Sonderband 18), Baden-Baden 2009, S. 351 - 384, S. 351.

8 Ebenda.

9 Vgl. Hubertus Buchstein, Reviving Randomness for Political Rationality, a.a.O. (Fn. 3), S. 450.

10 Vgl. ebenda; ders., Wählen, Losen und politische Gerechtigkeit, a.a.O. (Fn. 3).

11 Ders., Gehen Sie über Los! Das Zufallsprinzip als demokratisches Lebenselixier, in: Polar, Nr. 7 (2009), S. $41-44$. 
das Freud und Leid des Rentenkapitalismus und Immobilienmarktes zelebriert, den Familienfrieden für mindestens drei Stunden schwer beschädigt und zur asymmetrischen, irrationalen und ungerechten Verteilung des Mammons auf Grund des Zufalls, lies: des Würfelglücks führt. Jeder, der das schon einmal gespielt hat, weiß Bescheid: Mit einem mickrigen Bahnhof und der Bad- und Turmstraße als Besitz wird jede Runde in diesem Spiel zur Qual. Und wenn man immer nur der ist, der an die anderen zahlt, hofft man - wenn einen das Würfelglück ohnehin ignoriert - auf diese bekannte Ereigniskarte: „Gehe in das Gefängnis. Begib dich direkt dorthin. Gehe nicht über Los. Ziehe nicht 4.000 ein.“

Meine Damen und Herren, ganz ohne Umschweife, ich schätze diese Karte sehr und oute mich somit gerne als „Knastbruder“, also: Ich möchte nicht über Los gehen, und das bedeutet im übertragenen Sinne: Ich möchte dem Zufall nicht die Vorzüge zubilligen, die Hubertus Buchstein als gegeben ansieht, und ich möchte das vor allen Dingen nicht im Kontext einer repräsentativen, gewaltenteiligen Demokratie tun. ${ }^{12}$ Warum das so ist, bedarf natürlich der Begründung - insofern sei also die Aufforderung zur Auseinandersetzung mit Freude angenommen - eingedenk der schönen Mahnung in Dantes „Göttlicher Komödie“: „Und das sei dir immer Blei an den Füßen, damit du dich / Langsam wie ein Müder bewegst, bevor du ja oder nein sagst / Bei etwas, das du noch nicht verstanden hast."

Der angemessene Weg hierfür scheint mir zu sein, sich kurz der Problemdiagnose zu versichern, die dem Eintreten für die aleatorische Demokratie zu Grunde liegt, ebenso der Problemlösungsangebote, die die aleatorische Demokratie bereit hält und dann einige Kritikpunkte hierzu darzulegen.

\section{Aleatorische Demokratietheorie und Krisendiagnostik: Defizite des modernen Parlamentarismus}

Die Problemdiagnose, auf der die aleatorische Demokratietheorie fußt, zielt implizit und explizit auf die repräsentative Demokratie und ihre zentrale Institution beziehungsweise ihre zentralen Akteure: das Parlament und die Parteien. Kern dieser Diagnose ist ein zumindest partielles Auseinandertreten zwischen repräsentativer Demokratie beziehungsweise Repräsentation und parlamentarischer Demokratie. ${ }^{13}$ Vor diesem Hintergrund gerät auch das zentrale Verfahren, mit dem Herrschaft im Namen des Volkes delegiert wird, in ein schiefes Licht: die Wahl. Sie erscheint gerade hinsichtlich der Kontrolle der gewählten Amtsträger ungeeignet, den Volkswillen latent gegenüber sich verselbständigenden Parteiberufspolitikern zur Geltung zu bringen. Grundiert ist diese Problemdiagnose bei Buchstein allerdings

12 Es sei betont, dass dieser Konnex von Repräsentation und Gewaltenteilung für meine Ablehnung des Zufallsprinzips grundlegend ist. Vgl. für weitergehende Ausführungen: Roland Lhotta, Parlamentarismus als Gewaltenteilung. Institutionelle Ausprägungen komplexer Demokratie am Beispiel von Repräsentation, Regierungsbildung und Gesetzgebung, in: Helmar Schöne / Julia von Blumenthal (Hrsg.), Parlamentarismusforschung in Deutschland. Ergebnisse und Perspektiven 40 Jahre nach Erscheinen von Gerhard Loewenbergs Standardwerk zum Deutschen Bundestag, Baden-Baden 2009, S. 259 - 278.

13 Stilbildend und mit erheblicher Nachwirkung etwa die Diagnose bei Gerhard Leibholz, Repräsentativer Parlamentarismus und parteienstaatliche Demokratie, in: Kurt Kluxen (Hrsg.), Parlamentarismus, Königstein/Taunus 1980, S. 349 - 360, der einen krassen Gegensatz zwischen beiden konstruiert. 
mit neo-republikanischen Versatzstücken ${ }^{14}$, in denen Teilhabe an der Selbstregierung als Ausweis der politischen Freiheit nur dann funktioniert, wenn ihre Bürger diese politische Freiheit aktiv in Partizipation und Generierung des Gemeinwohls umsetzen und somit auch nach Ämtern streben beziehungsweise die Zuteilung von Ämtern als genauso selbstverständlich erachten wie die (zutiefst gleichheitliche) Vergabe dieser Ämter per Los. ${ }^{15}$

Dieses neo-republikanische Erbe wird aber von der aleatorischen Demokratietheorie recht speziell anverwandelt, denn im Unterschied zur Antike und zur besonders gern als Referenz benutzten athenischen Polis werden der Bürger und das ihm zugeloste Amt - oder gar auch die geloste Entscheidung - hier instrumentell vereinnahmt als Bestandteil einer korrektiven oder kompensatorischen Institutionenreform angesichts eines perzipierten Substanz- und Qualitätsdefizits repräsentativer Demokratie und damit auch des Parlamentarismus. ${ }^{16}$ Dieses Defizit wird ganz offenkundig mit den institutionalisierten Verfahren der repräsentativen Demokratie und dem Delegations- sowie Kontrollverhältnis zwischen Souverän und Repräsentanten in Verbindung gebracht: Die Verfahren scheinen nicht mehr darauf hinwirken zu können, dass die Repräsentanten so handeln, wie es normativ erwartet wird. Damit hängt die Evaluation der repräsentativen Demokratie natürlich davon ab, wie der normative Standard definiert wird. ${ }^{17}$ Diesen für die aleatorische Demokratietheorie zu rekonstruieren, ist nicht ganz einfach, weil Buchstein in seinem Plädoyer eine verschachtelte Montagetechnik verwendet, die im Ergebnis den Zufall als Agenten des Gemeinwohls in Gestalt von Rationalität, Gerechtigkeit und Gleichheit erscheinen lässt. Diesem Zufall jedenfalls, so zeige der Rekurs insbesondere auf die Antike und die italienischen Stadtrepubliken, habe man früher einmal mehr Vertrauen entgegengebracht; aktuelle Beispiele zur Anwendung des Zufalls aus der Geschäftsordnung des Bundestages oder zur Durchbrechung von Enthaltungen bei Bundesratsabstimmungen sowie vielfältige Beispiele aus dem anglo-amerikanischen und skan-

14 Hierzu David Held, Models of Democracy, Cambridge 2006, S. 29 ff.; John G. A. Pocock, The Macchiavellian Moment. Florentine Political Thought and the Atlantic Political Tradition, Princeton 1975; Cass Sunstein, Beyond the Republican Revival, in: Yale Law Journal, 97. Jg. (1988), S. 1539 - 1590; Frank Michelman, Law's Republic, in: Yale Law Journal, 97. Jg. (1988), S. 1493 - 1537; Michael J. Sandel, Democracy's Discontents: America in Search of a Public Philosophy, Cambridge 1996; Philip Pettit, Republicanism: A Theory of Freedom and Government, Oxford 1999.

15 Vgl. nur Benjamin R. Barber, Strong Democracy. Participatory Politics for a New Age, Berkeley 2003, besonders Kapitel 10.

16 Für einen prägnanten Überblick zum Diskussionsstand hierzu Heinrich Oberreuter, Substanzverluste des Parlamentarismus, in: APuZ, 62. Jg. (2012), H. 38/39, S. 25 - 31.

17 Es sei daran erinnert, dass es gerade in Deutschland zu Abwandlungen des Repräsentationsbegriffs gekommen ist, die daraus resultierten, dass dieser von einem Relations- zu einem Substanzbegriff geformt wurde. Dieser stand einem angemessenen Repräsentationsverständnis im Parlamentarismus entgegen, „weil mit dem substantiellen Abbilden des,Volkswillens' dem Parlamentarismus eine Repräsentationsaufgabe aufgebürdet wurde, die er organisatorisch nicht erbringen konnte und auch nicht sollte" (Oliver Lepsius, Repräsentation, in: Werner Heun / Martin Honecker / Martin Morlok / Joachim Wieland (Hrsg.), Evangelisches Staatslexikon, Stuttgart 2006, S. 2036 - 2041, S. 2038). Für eine immer noch wertvolle differenzierende Darstellung der Problematik vgl. Hasso Hofmann / Horst Dreier, Repräsentation, Mehrheitsprinzip und Minderheitenschutz, in: HansPeter Schneider / Wolfgang Zeh (Hrsg.), Parlamentsrecht und Parlamentspraxis in der Bundesrepublik Deutschland, Berlin / New York 1989, S. 165 - 197 sowie die leider zu wenig rezipierte Studie von Simona Rossi, La rappresentanza nello stato costituzionale. La rappresentazione degli interessi in Germania, Turin 2002. 
dinavischen Raum seien zudem Indikatoren für eine Renaissance des Zufalls in politischen Entscheidungsverfahren - so dass man sich ihm auch wieder zuwenden könne, zumal es manifesten Bedarf zur Korrektur partieller Defekte des parlamentarischen Systems gäbe.

Sein Plädoyer für eine experimentelle Institutionenreform pro aleatores montiert Buchstein in einer Mischung aus Neo-Republikanismus, deliberativer Demokratietheorie, Parlamentarismustheorie und -kritik, Theorien rationalen Handelns und Entscheidens sowie Repräsentationstheorie - um das mindeste zu nennen. Damit bewegt er sich gleichzeitig auf drei normativen Achsen der Demokratietheorie: Es geht um den Gegenstandsbezug, die Partizipationsintensität und das Rationalitätsniveau ${ }^{18}$ der modernen repräsentativen Demokratie. Entscheidend ist dabei der Umstand, dass das - normativ deduzierte - Argument für den aleatorischen Parlamentarismus sich nicht mit der Diagnose Schumpeters zu arrangieren vermag, Demokratie sei „die Herrschaft des Politikers“19. Und es ist ein Argument, das sich nicht mit dem Korrelat dieser Diagnose abfinden möchte: Dass es ein qua Repräsentation im Parlament zu vertretendes Gemeinwohl und einen Volkswillen, den es im Parlament (oder ersatzweise woanders!) zu „repräsentieren“ gelte, ebenfalls nicht gibt. ${ }^{20}$

Dies wird deutlich, wenn man die Rekonstruktion des liberalen Erbes des modernen Parlamentarismus und der Repräsentation durch Buchstein näher betrachtet. Hier werden vier klassische Begründungen für die repräsentative Demokratie aufgelistet und mit den üblichen „Verdächtigen“" annotiert:

- Elitenauslese;

- funktionale Arbeitsteilung, bei der Berufspolitiker in einer immer komplexeren Welt als professionelle Spezialisten für die Herstellung des allgemeinen Willens zuständig sind;

- Herstellung rationaler Entscheidungen durch Rede und Gegenrede und den Austausch von Argumenten auf der Basis hinreichender Information und „preference laundering“;

- Heterogenität der Repräsentanten, die das Entstehen stabiler Mehrheiten und einer Tyrannei der Mehrheit verhindert und damit Minderheiten schützt beziehungsweise Entscheidungen generiert, die Toleranz und Pluralismus befördern. ${ }^{21}$

Alle vier Begründungen sind den Ausführungen Buchsteins zufolge bis heute der Kernbereich für die Rechtfertigung des modernen Parlamentarismus geblieben. ${ }^{22}$ Allein, im Lichte der Entwicklung moderner Massendemokratien und der Erfahrungen mit der Praxis und Performanz parlamentarischer Systeme hätten alle vier Begründungen signifikant an Überzeugungskraft verloren und seien durch die Realität überholt oder zumindest teilweise widerlegt und substituiert. Die andauernde Debatte über angemessene Reformen moderner parlamen-

18 Vgl. Hubertus Buchstein, Demokratie, in: Gerhard Göhler / Mattias Iser / Ina Kerner (Hrsg.), Politische Theorie. 25 umkämpfte Begriffe zur Einführung, Wiesbaden 2011, S. 46 - 62, S. 50 ff.

19 Joseph A. Schumpeter, Kapitalismus, Sozialismus und Demokratie, Bern 1950, S. 452.

20 Vgl. ebenda, S. 373 ff., insbesondere S. $400 \mathrm{ff}$.

21 Vgl. Hubertus Buchstein, Elective and Aleatory Parliamentarism, a.a.O. (Fn. 3), S. 7 ff.

22 Ich finde es bemerkenswert, dass Buchstein ein anderes zentrales Element aus diesem Kanon nicht nennt: das freie Mandat. Dies, obwohl es doch konstitutiv für die Vertretungsrolle des Repräsentanten ist und seit Sieyès und Burke zu den definitorischen Grundelementen für Freiheit und Rollenverständnis des Abgeordneten in der parlamentarischen Demokratie gehört. Vgl. nur Stefan Marschall, Parlamentarismus. Eine Einführung, Baden-Baden 2005, S. 34 ff. Dies gilt umso mehr, als es gerade das freie Mandat - in Kombination mit dem Typus des Berufsparteipolitikers - ist, das den Weg zu dem ebnet, was Buchstein unter „Neutralitätsdefizite“ rubriziert und zum Auslöser für den Einsatz des Korrektivs „Zufall“ in Gestalt eines „House of Lots“ macht. 
tarischer Systeme seien ein Indikator für die Unzufriedenheit mit ihrer Leistungsfähigkeit. ${ }^{23}$ Ein Teil der Literatur sekundiert hier gern. Das Narrativ hierzu - und hier erlaube ich mir ein wenig Zuspitzung und Vereinfachung - geht ungefähr so:

Erstens: Elitenauslese ist eigentlich nur noch Parteielitenauslese, nicht eine die ganze Gesellschaft umfassende Bestenauslese - die Lektionen von Robert Michels und Joseph Schumpeter haben Parteiensysteme und die von ihnen getragenen repräsentativen Demokratien offenkundig gründlich ausbuchstabiert. Wahlsysteme, in denen Wahlkreisbindung und Direktmandate zu Gunsten von parteiengesteuerten Listen zurückgedrängt werden, bieten den institutionellen Kontext hierfür, ebenso wie Entwicklungen der Parteiensysteme zu Regierungsmehrheitsproduzenten, was unweigerlich in eine Frontstellung von Repräsentativität und Responsivität führe und die Legitimitätsgrundlagen moderner repräsentativer Demokratien und ihrer Parteien erodiere. ${ }^{24}$ Parteidisziplin gehe dann allemal vor rational-vernünftigen Problemlösungen und Gemeinwohlorientierung.

Zweitens: Die funktionale Arbeitsteilung im Parlament ist ein parteipolitisch vermitteltes Funktionselitensystem von Berufspolitikern, die sich vom Volkssouverän und vom Volkswillen abkoppeln, was wiederum zu einer Entkopplung von repräsentativer und parlamentarischer Demokratie führt.

Drittens: Der Verlust des „deliberative spirit“: Rationale, vernünftige Entscheidungen zum Wohle aller oder der allermeisten, hervorgegangen aus Rede und Gegenrede - das war schon immer ein demokratietheoretisch-deliberativer Traum. Im macht- und parteipolitischen Konkurrenzkampf ist er endgültig zerstoben. Reden und Redezeit werden durch parteipolitische Funktionseliten im Parlament gesteuert, Plenarreden sind Fensterreden, und Entscheidungen sind weder rational noch vernünftig noch transparent, sondern verlagern sich zunehmend in black boxes wie den Koalitions- und Vermittlungsausschuss ${ }^{25}$ oder werden gleich gouvernemental präkludiert, wie das Bundesverfassungsgericht in den letzen Jahren wiederholt bemängelt hat. ${ }^{26}$

Viertens: Der schöne Traum einer möglichst breiten Repräsentation der Bevölkerung im Parlament bricht sich regelmäßig an der asymmetrischen Verteilung von Mandaten und politischem Engagement hinsichtlich der Berufs- und Statusgruppen im Parlament. „Mirror representation" gab es früher schon nicht und gibt es jetzt auch nicht. Das gleiche und freie Wahlrecht für alle, das historisch in langwierigen Kämpfen ertrotzt werden musste, führte zwar in die Massendemokratie, aber nicht in die Repräsentation der Massen, sondern in den oligopolistischen Parteienstaat. Langfristig befördere dieser wiederum Politikverdrossenheit, sinkende Wahlbeteiligung sowie einen Rückgang der Bindungskraft der Parteien. ${ }^{27}$

23 Vgl. Hubertus Buchstein, Elective and Aleatory Parliamentarism, a.a.O. (Fn. 3), S. 7.

24 Vgl. Peter Mair, Representative versus Responsible Government, MPIfG Working Paper 09/8, Köln 2009; ders., The Challenge to Party Government, in: West European Politics, 31. Jg. (2008), H. 1/2, S. $211-234$.

25 Vgl. Wolfgang Rudzio, Das politische System der Bundesrepublik Deutschland, Wiesbaden 2011, S. 267 ff.; Roland Lhotta, Konsens und Konkurrenz in der konstitutionellen Ökonomie bikameraler Verhandlungsdemokratie: Der Vermittlungsausschuss als effiziente Institution politischer Deliberation, in: Everhard Holtmann / Helmut Voelzkow (Hrsg.), Zwischen Wettbewerbs- und Verhandlungsdemokratie. Analysen zum Regierungssystem der Bundesrepublik Deutschland, Wiesbaden 2000, S. 79 - 103.

26 Vgl. 2 BvE 2/08; 2 BvE 4/11; 2 BvE 8/11; 2 BvR 987/10; 2 BvR 1099/10; 2 BvR 1485/10.

27 Vgl. Klaus Detterbeck, Parteien und Parteiensystem, Konstanz 2011, S. 128 ff. 


\section{Die präsumtiven Vorteile des Zufalls und seine Institutionalisierung im „House of Lots“}

Hier verspricht die aleatorische Demokratietheorie nun frischen Wind in die schlaffen und ausgefransten Segel der repräsentativen Demokratie zu pusten und die alte Fregatte an bessere Gestade zu steuern, wo der Zufall in Gestalt von Losverfahren waltet. Fünf Argumente werden hierfür ins Feld geführt ${ }^{28}$ :

Erstens: Losverfahren würden die Maximierung des Gleichheitsprinzips hinsichtlich der Erfolgschancen eines jeden Individuums ermöglichen. Sie seien deswegen geeignet für Demokratien, wenn es um die Verteilung knapper Güter und Ressourcen geht, aber auch um die Verteilung von Ämtern, Aufgaben etc.

Zweitens: Losverfahren könnten in regelmäßigen Abständen wiederholt werden, um zum Beispiel Ämter zu besetzen - damit bliebe die Chance für alle gleich hoch, in ein Amt zu gelangen oder auch davon verschont zu bleiben, was wiederum Stabilität und Akzeptanz generiere.

Drittens: Losverfahren können entlastend wirken - sowohl für Entscheidungsträger als auch für Entscheidungsadressaten; dies sei gerade mit Blick auf knappe Güter und Ressourcen eine attraktive Option.

Viertens: Losverfahren seien ein neutraler und autonomer Zufallsmechanismus zur Generierung von Entscheidungen - das mache sie geeignet als „tie breaker“ zur Indifferenzregulation und Kompromisserzwingung in Blockadesituationen. ${ }^{29}$

Fünftens: Losverfahren generieren Ergebnisse, die überhaupt nicht vorbestimmt sind. Dies könne ein wirksames Mittel sein, um Korruption und Einflussnahme im Vorfeld von Entscheidungen einzudämmen, denn es gäbe keine mandatierten Entscheider; ihre Bearbeitung durch Parteien oder Lobbyisten wäre damit irrelevant.

Wenn wir für den Augenblick konzedieren, dass dies plausible oder zumindest bedenkenswerte Argumente für den „Zufall mit Absicht“ sind, stellt sich sofort die Frage nach dem Verbindlichkeitsstatus zufallsgenerierter Entscheidungen sowie der Legitimität zugeloster Ämter, von denen solche Entscheidungen gefällt werden. Und damit sind wir auch gleich bei den beiden entscheidenden Problemen hinsichtlich institutioneller Experimente mit der Aleatorik im Rahmen einer repräsentativen Demokratie: Was wäre der Status von institutionalisierten Losverfahren beziehungsweise Losentscheidungen im institutionellen Design des parlamentarischen Bundesstaates der Bundesrepublik? Und was wären mögliche Sachbereiche beziehungsweise Politikfelder, für die Losverfahren besonders in Frage kämen.

Hubertus Buchstein hat auch hierzu einen konkreten Vorschlag gemacht, der sich um das institutionelle Novum eines sogenannten „House of Lots“ dreht. Aus seiner Sicht sprechen in den allermeisten Fällen „gute Argumente dafür, einer Versammlung von gewählten Berufspolitikern wie dem Deutschen Bundestag die politische Entscheidungsmacht zu überantworten. Bei solchen Entscheidungsmaterien allerdings, bei denen davon ausgegangen werden muss, dass diese Politiker (oder zumindest die Mehrheit von ihnen) aufgrund eigener machtpolitischer oder materieller Interessen befangen sind, sollten wir nach einem anderen Weg suchen, um zu einer der politischen Gerechtigkeit und Fairness verpflichteten Entscheidung zu 
gelangen“30. Allgemeiner formuliert - und hier sei nochmals ein Zitat erlaubt: „Bei der Suche nach einem gerechten Verfahren sollten wir als dritten institutionellen Weg in all den Fällen, in denen es klare Anhaltspunkte für ein Neutralitätsdefizit (auf Seiten der politischen Eliten) sowie zusätzlich für ein Willensdefizit (auf Seiten der Bevölkerung) gibt, die Möglichkeit nutzen, die politische Entscheidung einem temporären ,House of Lots' nach dem Muster der Citizen Assembly zu überantworten. "31

Ein solches House of Lots sei eine institutionelle Alternative zu Kommissionen wie Gerichten und könnte im institutionellen Kontext moderner Demokratien als eine demokratisch-deliberative Version jener pouvoir neutre anverwandelt werden, die Benjamin Constant und Carl Schmitt schon so erstrebenswert fanden. ${ }^{32}$

\section{Der Zufall als Agent des Guten und Richtigen}

Damit, meine Damen und Herren, wäre nun der Punkt gekommen, an dem die eine oder andere Kritik an Hubertus Buchsteins Ausführungen fällig ist. Zu diesem Zwecke möchte ich zunächst daran erinnern, dass „eine prästabilierte Harmonie von Wille und Norm“ dem liberal-demokratischen Verfassungsstaat fremd ist: „Seine Freiheitlichkeit widerstreitet jeder Gemeinwohldefinition, auch der demokratischen. Das Gute und Richtige bleibt auch nach der verbindlichen Entscheidung offen und diskutabel. "33 Warum dieses Zitat? Weil der gesamte „neo-athenische“ Introitus zu Buchsteins Plädoyer für das Losverfahren in seiner Begründung für das House of Lots auf einen zentralen Aspekt zusammenschnurrt, der oben bereits angedeutet wurde: Die Nichtakzeptanz einer als normativ unbefriedigend empfundenen Leistung von Berufspolitikern im Rahmen eines Delegationsverhältnisses, dessen normativer Repräsentationsgehalt entleert und dessen Kontrollintensität seitens des Volkes schwach sei. Der Zufall wird bei Buchstein somit zum Agenten des Guten und Richtigen denn es sollen doch politische Entscheidungen zustande kommen, die gerecht und fair sind. Das aber sei nicht möglich, wenn Politiker bei ihren Entscheidungen machtpolitischen und materiellen Interessen folgen würden. Und hier entfaltet die Kontrastierung der Realität mit den vier Funktionen von Repräsentation und ihrem normativen Anspruch auf Verwirklichung ihre ganz eigene Wirkung, speziell hinsichtlich des konstatierten Verlustes an Deliberation. Denn gerade diesen möchte Buchstein nicht akzeptieren und führt deshalb die deliberative Komponente mittels eines zufallsgenerierten House of Lots wieder ein: Hier werden dessen ausgeloste, temporär und problembezogen tätige Mitglieder in die Lage versetzt, sich deliberativ einen informierten politischen Willen zu bilden, der die Gewähr bietet, rationaler, gerechter, fairer zu sein, mithin: dem Gemeinwohl und dem Volkswillen näher zu kommen und damit repräsentativer zu sein als die neutralitätsdefizitären Parteipolitiker im Parlament und die willensdefizitären Median-Bürger. Damit passiert hier argumentativ ziemlich

30 Hubertus Buchstein, Wählen, Losen und politische Gerechtigkeit, a.a.O. (Fn. 3), S. 406 (Hervorhebung des Verfassers).

31 Ebenda, S. 405 (Hervorhebungen des Verfassers).

32 Vgl. ders., Elective and Aleatory Parliamentarism, a.a.O. (Fn. 3), S. 12.

33 Hans-Detlef Horn, Demokratie, in: Otto Depenheuer / Christoph Grabenwarter (Hrsg.), Verfassungstheorie, Tübingen 2010, S. 743 - 776, S. 760, Rz. 40. 
genau das, was Schumpeter den „utilitaristischen Vätern der demokratischen Lehre“ ins Stammbuch schrieb:

„Sie leiteten ihren Volkswillen ganz einfach vom Willen der Individuen ab. Sofern es keinen Mittelpunkt - das Gemeinwohl - gibt, nach dem wenigstens auf die Dauer alle individuellen Willen gravitieren, werden wir nicht jenen besonderen Typ der ,natürlichen' volonté générale erhalten. Das utilitaristische Gravitationszentrum vereinheitlicht einerseits die individuellen Willen und tendiert darnach, sie vermittels rationaler Diskussion in den Willen des Volkes einzuschmelzen; andererseits verleiht es dem letzteren diese exklusive sittliche Würde, wie sie vom klassischen demokratischen Glaubensbekenntnis gefordert wird. Dieses Glaubensbekenntnis besteht nicht einfach darin, den Willen des Volkes als solchen zu verehren, sondern beruht auf gewissen Annahmen über das ,natürliche' Ziel dieses Willens - ein Ziel, das durch die utilitaristische Vernunft sanktioniert ist. Sowohl die Existenz wie auch die Würde dieser Art von volonté générale verschwinden, sobald uns die Vorstellung eines Gemeinwohls fehlt. Und beide Stützen der klassischen Lehren zerbröckeln unweigerlich zu Staub. “34

Damit wir uns nicht missverstehen: Natürlich haben normative Maßstäbe bei der Evaluation eines politischen Systems ihre vollste Berechtigung - so sind die Fragen nach einer guten und gerechten Ordnung in der Politikwissenschaft und damit auch ihre Charakteristik als praktische Philosophie in den letzten Dekaden mit viel zu leichter Hand verabschiedet worden. ${ }^{35}$ Aber die parlamentarische Demokratie schützt nun einmal nicht vor Entscheidungen, Belastungen und Abhängigkeiten, die sich aus dem Aufeinandertreffen staatlicher Herrschaft in Gestalt mandatierter Amtsträger und Bürgern als Herrschaftsadressaten ergibt. Sie unterwirft die Herrschaftsgewalt zunächst einmal lediglich der „Abhängigkeit von der Volksbeurteilung " in regelmäßigen Abständen. ${ }^{36}$ Das scheint für Buchstein aber ein demokratie- und repräsentationstheoretischer Diminutiv zu sein, wie sein Blick auf die von ihm ausgemachten vier klassischen Funktionen - insbesondere Deliberation - zeigt, angesichts derer jede institutionelle Verwirklichung der repräsentativen Demokratie in eine evaluative Einbahnstraße gerät. Die Geschichte der Parlamentarismuskritik zeigt hinlänglich, dass dies ein bewährtes Muster ist, und niemand hat das wohl besser beherrscht, als der vorhin bereits als Referenz für die pouvoir neutre bemühte Carl Schmitt. Seine berühmte Parlamentarismuskritik von $1926^{37}$ entwirft ein ideengeschichtlich rekonstruiertes Reinbild des Parlamentarismus, an dem schon damals die Realität der liberalen Demokratie und des parteiendominierten Parlamentarismus scheitern musste und sollte. Übertragen auf unsere Problemstellung: Das Operieren mit normativ deduzierten Maßstäben, die puristisch und erstaunlich selbstgewiss hinsichtlich der tauglichen Mittel und Bestandteile von Repräsentation sowie der Existenz beziehungsweise Herstellbarkeit eines zu repräsentierenden (informierten) politischen Willens des Volkes sind, taugt nur begrenzt als Grund für experimentelle institutionelle Reformen an einem System, dessen implizite Logik erst einmal erfasst sein muss. Dies

34 Joseph A. Schumpeter, a.a.O. (Fn. 19), S. 401 (Hervorhebungen im Original).

35 Es sei hier gern erinnert an Wilhelm Hennis, Politik und praktische Philosophie. Eine Studie zur Rekonstruktion der politischen Wissenschaft, in: ders., Politikwissenschaft und politisches Denken, Tübingen 2000, S. 1 - 126; vgl. auch die Beiträge von Peter Graf Kielmansegg, Tine Stein, Reinhard Mehring und Andreas Anter, in: ders. (Hrsg.), Wilhelm Hennis' Politische Wissenschaft, Tübingen 2013.

36 Vgl. Hans-Detlef Horn, a.a.O. (Fn. 33), S. 762, Rz. 45.

37 Vgl. Carl Schmitt, Die geistesgeschichtliche Lage des heutigen Parlamentarismus, Berlin 1991 (unveränderter Nachdruck der 1926 erschienenen 2. Auflage). 
gilt meines Erachtens leider auch für Buchsteins institutionelle Triangulation von Deliberation, Rationalität und Zufall in der repräsentativen Demokratie.

\section{Repräsentation, Bürger, Staat und Herrschaft: Eine Erinnerung an das Amt und die Gewaltenteilung}

Und damit bin ich bei meinem nächsten Kritikpunkt: Ich teile nicht die Ansicht, dass Losverfahren ohne Weiteres kompatibel mit der institutionellen Logik eines parlamentarischen Systems beziehungsweise einer repräsentativen Demokratie sind. Und ich teile auch nicht die Auffassung, dass sie die Rationalität politischer Entscheidungsprozesse steigern können. Der entscheidende Grund hierfür liegt darin, dass Zufall und Amt in einer repräsentativen Demokratie nicht institutionell zusammengespannt werden können und sollten. ${ }^{38}$ Der Annahme, dass dies geht, kann man indessen nur anheimfallen, wenn man - und hier ist die zweite demokratie- und repräsentationstheoretische Diminutivdiagnostik bei Buchstein zu verorten - das Wahlamt einer Kontroll- und Korrektivlogik unterwirft, in der die Lostrommel im Namen des Gemeinwohls (in Gestalt von Gerechtigkeit, Fairness usw.) den Ersatz für imperatives Mandat, Recall und direkte Demokratie spielt - weil sowohl Berufspolitiker als auch (Wahl-) Bürger anscheinend nicht wissen, was sie wollen sollen. ${ }^{39}$ Aus einer Perspektive, die an ein repräsentierbares Gemeinwohl glaubt und Partizipation über den Wahlakt hinaus als intrinsischen Wert begreift, ist dies ein institutionell zu kompensierendes „Leiden an Unbestimmtheit“ 40 . Da zudem in der modernen parlamentarischen Demokratie der „internalistische Moralismus “ ${ }^{41}$ des in seinem Handeln auf das Gemeinwohl bezogenen Einzelnen gleich doppelt zerschellt - beim Berufspolitiker als Amtsträger an seinen Neutralitätsdefiziten, beim Wahlbürger an seinen Willensdefiziten - bedarf es hier eines Remediums beziehungsweise einer „Perfektionsagentur“ ${ }^{“ 2}$. Und genau hier ist der Ort, an dem der institutionalisierte Zufall die defekte Repräsentation in der modernen parlamentarischen

38 Irritierend ist Buchsteins in Abschnitt 7 seines Beitrags formulierte Auffassung, hierbei handele es sich um eine "vormoderne“ Auffassung vom Amt, die in der Verbindung mit der modernen Demokratietheorie auf die legitimationstheoretische Metapher von den ungebrochenen demokratischen Legitimationsketten zuführe. Denn jemand, der Neutralitäts- und Willensdefizite beklagt, rasselt selbst mit diesen Legitimationsketten und erweist sich als Mitglied einer "chain gang“, die dem Amtsträger und seinem Wahlvolk situativ sogar besonders kurze Ketten schmiedet - davon lenkt die konzeptuelle Dispersion von Verantwortlichkeit auf partiale Mechanismen der Verantwortlichkeitsorganisation unter Berufung auf Keohane / Nye / Grant nur unzulänglich ab.

39 Es ist dies wohl dem Versuch geschuldet, den globalen Trend zur Rationalisierung von Demokratie beziehungsweise demokratischer Entscheidungsprozesse sowie die damit einher gehende prozessorientierte Output-Fixierung mit dem intrinsischen Wert von politischer Partizipation als zu bewahrendem Versprechen der Demokratie zu versöhnen. Vgl. die in dieser Hinsicht bezeichnenden Ausführungen in: Hubertus Buchstein, After Athens - The Genealogy of Modern Democracy, in: Redescriptions, 10. Jg. (2006), S. 150 - 159, S. 159.

40 Axel Honneth, Leiden an Unbestimmtheit. Eine Reaktualisierung der Hegelschen Rechtsphilosophie, Stuttgart 2001.

41 Wolfgang Kersting, Jean-Jacques Rousseaus „Gesellschaftsvertrag“, Darmstadt 2002, S. 68.

42 Ders., Die Vertragsidee des Contrat Social und die Tradition des neuzeitlichen Kontraktualismus, in: Reinhard Brandt / Karlfriedrich Herb (Hrsg.), Vom Gesellschaftsvertrag oder Prinzipien des Staatsrechts, Berlin 2000, S. 45 - 66, S. 60. 
Demokratie optimieren soll ${ }^{43}$ - und sich gleichzeitig ein Kompatibilitätsproblem ergibt. Vergegenwärtigen wir uns kurz, worum es bei der Repräsentation in der parlamentarischen Demokratie eigentlich geht:

In der klassischen Definition von Ernst Fraenkel bedeutet Repräsentation ${ }^{44}$ „die rechtlich autorisierte Ausübung von Herrschaftsfunktionen durch verfassungsmäßig bestellte, im Namen des Volkes, jedoch ohne dessen bindenden Auftrag handelnde Organe eines Staates oder sonstigen Trägers öffentlicher Gewalt, die ihre Autorität mittelbar oder unmittelbar vom Volk ableiten und mit dem Anspruch legitimieren, dem Gesamtinteresse des Volkes zu dienen und dergestalt dessen wahren Willen zu vollziehen“" ${ }^{45}$.

Hier wird mehr als deutlich, dass Repräsentant zu sein, eben nicht bedeutet, repräsentativ zu sein. Die Ausübung von Herrschaft ist in der repräsentativen Demokratie Herrschaft im Namen des Volkes; sie ist nicht an einen Auftrag gebunden; und diese Herrschaft hat lediglich den Anspruch, dem Gesamtinteresse des Volkes zu dienen. Sie leitet ihre Autorität - ihr Amt - deswegen aber vom Volk ab. Ideengeschichtlich signalisiert diese Herrschaftsableitung die repräsentationstheoretisch bedeutungsvolle Etablierung eines neuen Konzepts des Bürgers: „By the time representative government arose, the kind of political equality that was at center stage was the equal right to consent to power, and not - or much less so - an equal chance to hold office. This means that a new conception of citizenship had emerged: citizens were now viewed primarily as the source of political legitimacy, rather than as persons who might desire to hold office themselves." 46

Man kann das mit Manin so interpretieren, dass dies in der modernen (repräsentativen) Demokratie dazu geführt habe, den Bürger immer weniger als jemanden zu betrachten, der nach einem Amt strebt, wodurch wiederum der Blick dafür verloren ging, wie Ämter als knappe Güter von repräsentativen Institutionen an die Bürger verteilt werden. ${ }^{47}$ Man kann es mit Sartori aber auch so verstehen, dass „die Mikrodemokratie der Antike im Unterschied zur modernen Makrodemokratie nicht vor dem Problem der Beziehung zwischen Bürger und Staat stand“48. Das sind zwar zwei Seiten derselben Medaille - aber die Sartori-Seite bedingt die Manin-Seite, und das ist bedeutsam, denn daraus ergibt sich, dass der umfängliche Rückgriff Buchsteins auf die Antike möglicherweise nur bedingt dazu taugt, einem „aleatorischen Parlamentarismus“ argumentativ den Boden zu bereiten, weil sie genau diese (Herrschafts-)Beziehung Staat-Bürger in der repräsentativen Demokratie verkennt. Demokratie, zumal repräsentative Demokratie ist im modernen Staat nämlich eine Form der Herrschaftsorganisation, mit der die Herrschaftssubjektivität des Staates und seiner Macht gegenüber den Freiheitsansprüchen seiner Bürger dadurch erträglich gemacht wird, dass sie

43 Das hier zu Grunde liegende Problem einer institutionellen Hegung individueller Freiheit wird ausführlicher behandelt bei Roland Lhotta, Der antiliberale Kern des neuen Institutionalismus in der Politikwissenschaft: Überlegungen zum aktuellen Erscheinungsbild der Disziplin, in: ZPol, 16. Jg. (2006), H. 1, S. 7 - 28.

44 Vgl. im Übrigen allgemein Hasso Hofmann, Repräsentation. Studien zur Wort- und Begriffsgeschichte von der Antike bis zum 19. Jahrhundert, Berlin 1998; Hanna F. Pitkin, The Concept of Representation, Berkeley 1967.

45 Ernst Fraenkel, Deutschland und die westlichen Demokratien, Frankfurt am Main 1990, S. 153.

46 Bernard Manin, a.a.O. (Fn. 6), S. 92.

47 Vgl. ebenda, S. 93.

48 Giovanni Sartori, Demokratietheorie, Darmstadt 1992, S. 289. 
auf die Volkssouveränität zurückgeführt wird. ${ }^{49}$ Deswegen gibt es Wahlen, und deswegen werden mit Herrschaftsmacht ausgestattete Ämter mit Personen besetzt, die gewählt werden und ihr Tun zu verantworten haben. Die Herrschaftsbeziehung Staat-Bürger stattet das Amt aber mit einer völlig anderen institutionellen Logik aus, als sie das zugeloste Amt in der athenischen Demokratie hatte.

Diese Logik führt natürlich sofort zu der für jedes Repräsentationsverhältnis zentralen Problematik von Delegation und Herrschaft. Vom Prinzipal durch die Wahl betraute Agenten üben Herrschaft aus, die akzeptabel und kontrollierbar gemacht werden muss, um nicht in eine „bloße Machtbeziehung "50 oder eine durch den Wahlakt autorisierte Oligarchie ${ }^{51}$ abzugleiten. Dabei geht es sowohl um prozedurale Elemente der Kontrolle als auch um den angemessenen Kontrollmaßstab: Die Wahl scheint als Kontrollverfahren unzulänglich, weil sie die gewählten Amtsträger an einer viel zu langen Kette führt und diese ihren Neutralitätsdefiziten frönen können. Und der (einheitliche und/oder authentische) Volkswille ist entweder eine Erfindung, oder aber er kommt nicht zustande, weil das Volk Willensdefizite hat - damit fällt er als Kontrollmaßstab quasi aus. Das sich hieraus ergebende Kontrolldilemma ist der zentrale Ansatzpunkt der aleatorischen Demokratietheorie - denn der Einsatzbereich des von Buchstein vorgeschlagenen House of Lots zielt auf solche Entscheidungssituationen, in denen präsumtiv bloße Machtbeziehungen dominieren, weil beim Mandatsträger Neutralitätsdefizite vorliegen oder aber der Prinzipal ein Willensdefizit hat und damit seinen wahren Willen nicht zu vermitteln vermag. ${ }^{52}$ Wer übrigens definiert, wann das der Fall ist, bleibt im Dunkeln - aus dem sich hoffentlich niemals ein aleatorischer Wohlfahrtsausschuss als Präzeptor schwankend erhebt. ${ }^{53}$ Die Formulierungen von Buchstein lassen trotzdem aufhorchen: „In all den Fällen, in denen es klare Anhaltspunkte für ein Neutralitätsdefizit (auf Seiten der politischen Eliten) sowie zusätzlich für ein Willensdefizit (auf Seiten der Bevölkerung) gibt“ - wer auch immer darüber befindet: Soll das tatsächlich die Schwelle für den Einsatz des Zufalls beziehungsweise die situative Aktivierung eines durch den Zufall generierten House of Lots sein? Denn mit Willens- und Neutralitätsdefiziten wird nicht ein Grenzfall, sondern der Normalfall in einer repräsentativen Demokratie

$49 \mathrm{Vgl}$. Hans-Detlef Horn, a.a.O. (Fn. 33), S. $763 \mathrm{ff}$.

50 Werner J. Patzelt, Vergleichende Parlamentarismusforschung als Schlüssel zum Systemvergleich. Vorschläge zu einer Theorie- und Forschungsdebatte, in: Winfried Steffani / Uwe Thaysen (Hrsg.), Demokratie in Europa. Zur Rolle der Parlamente, Opladen 1995, S. 355 - 385, S. 374 f.

51 Vgl. Nadia Urbinati, Representative Democracy: Principles and Genealogy, Chicago 2006, S. 15.

52 Hier ist es unverständlich, dass Buchstein sich gegen den Vorrang eines „angeblich authentischen Volkswillens" im Plebiszit ausspricht, weil, notabene, ein solcher Wille empirisch in den seltensten Fällen vorliege. Dies gelte zumal für Wahlrechtsfragen, zu denen ein informierter politischer Wille kaum identifizierbar sei. Das Problem ist nur, dass der informierte politische Wille, um den es Buchstein zu tun ist, ja durch Rationalität und Deliberation befördert werden soll und genau dieser Wille dann substitutiv für den ,angeblich authentischen Volkswillen“ fungiert, dabei aber dessen Richtigkeits- und Verbindlichkeitsanspruch übernimmt. Dies kann auch gar nicht anders sein, denn dieser Wille hat ja die „Perfektionsagentur" des ausgelosten, rational-deliberativ entscheidenden House of Lots durchlaufen und kommt somit dem authentischen Volkswillen am nächsten.

53 Die Frage pressiert aber, denn sowohl die neutralitätsdefizitären Abgeordneten/Amtsträger als auch die willensdefizitären Wahlbürger dürften in einer solchen Situation je für sich nicht in der Lage sein, diese Defizite zu erkennen und ihre präsumtiv defizitären Entscheidungen deshalb an eine zufallsgenerierte „Perfektionsagentur“ wie das House of Lots zu delegieren. 
beschrieben. Willensdefizite des Volkes sind geradezu konstitutiv für eine repräsentative Demokratie - sonst könnten wir ja alles selbst entscheiden. Und Neutralitätsdefizite sind zwar ein Ärgernis, aber auch eine Triebkraft des Parteienwettbewerbs in der repräsentativen Demokratie $^{54}$ und ein Differenzierungs- und Evaluierungskriterium bei der nächsten Wahl.

Für den Ausgleich und die Kompensation dieser Defizite haben repräsentative Demokratien indessen an der Schnittstelle der Herrschaftsbeziehung zwischen Staat und Bürger etwas geschaffen, das man in der schönen Begriffsprägung von James G. March / Johan P. Olsen als „integrative Institution" ${ }^{55}$ bezeichnen kann: das Amt. In dieses wird man gewählt und/oder im Namen Aller berufen. Gerade repräsentative Demokratien versehen Ämter mit Werten und Handlungslogiken, die auf eine „ethic of public identity and service“56 zielen und die Leistung der Amtsinhaber in dieser Hinsicht öffentlich kontrollierbar und verantwortbar macht ${ }^{57}$, um den für Repräsentation und das „Amt“ notwendigen Konsens immer wieder neu herzustellen. Denn dieser ist „in der repräsentativen Demokratie auf die Ausübung von Ämtern bezogen, und zwar in einer Weise, daß sie das durch die Berufung in das Amt bekundete Vertrauen - unter ständiger Kontrolle - rechtfertigt. Die Verantwortlichkeit besteht darin, daß man sich der Kritik für die Amtsführung stellt und gegebenenfalls die Konsequenzen durch Nichtwiederwahl oder Entlassung tragen muß ${ }^{\text {"58 }}$.

Repräsentation erfordert demnach ein komplexes „Arrangement von vielerlei Institutionen und funktional verschränkten Prozessen“59, um Herrschaft zu limitieren und zur Verantwortung zu ziehen, also auch: ein komplexes System der Gewaltenteilung ${ }^{60}$, das die multiple (!) Repräsentation eines de facto pluralen und nur zurechnungstechnisch einheitlichen Volkswillens und dessen organschaftliche und prozedurale Abbildung ermöglicht durch integrative Institutionen wie das Amt, die als „,embodiment and instrument of the community, or of the democratic order as a constitutional system " 61 fungieren, dabei aber durchaus widerstreitende Handlungslogiken aufnehmen. Mit anderen Worten: Repräsentation und Gewaltenteilung sind in der parlamentarischen Demokratie koinzident, Parlamentarismus ist immer auch Gewaltenteilung ${ }^{62}$ - und eine „pouvoir neutre“ braucht dieses System schon allein deswegen nicht, weil es um eine bewusste und gewollte Verschränkung konkurrierender Handlungslogiken geht, deren „neutralisierende“ Überspielung durch den

54 Vgl. nur die Einschätzung bei Joseph A. Schumpeter, a.a.O. (Fn. 19), S. 452 f.

55 James G. March / Johan P. Olsen, Institutional Perspectives on Governance, in: Hans-Ulrich Derlien / Uta Gerhardt / Fritz W. Scharpf(Hrsg.), Systemrationalität und Partialinteresse. Festschrift für Renate Mayntz, Baden-Baden 1994, S. 249 - 270, S. 264 ff.; dies., Rediscovering Institutions. The Organizational Basis of Politics, New York / London 1989, S. 124 ff.

56 Dies., Institutional Perspectives on Governance, a.a.O. (Fn. 55), S. 265.

57 Vgl. dies., Democratic Governance, New York u.a. 1995, S. 57 ff.

58 Wilhelm Hennis, Amtsgedanke und Demokratiebegriff, in: ders., Politikwissenschaft und politisches Denken, Tübingen 2000, S. 127 - 147, S. 137.

59 Werner J. Patzelt, a.a.O. (Fn. 50), S. 374.

60 Dies ist eine der wichtigsten und bleibenden Erkenntnisse der Federalist Papers. Vgl. hierzu Roland Lhotta (Hrsg.), Die hybride Republik. Die Federalist Papers und die politische Moderne, BadenBaden 2010; aber auch die Ausführungen bei Ingeborg Maus, Volkssouveränität und ,Repräsentation' oder: ein Aspekt der Gewaltenteilung, in: dies., Zur Aufklärung der Demokratietheorie. Rechts- und demokratietheoretische Überlegungen im Anschluß an Kant, Frankfurt am Main 1994, S. $191-202$.

61 James G. March / Johan P. Olsen, Rediscovering Institutions, a.a.O. (Fn. 55), S. 126.

62 Vgl. Roland Lhotta, a.a.O. (Fn. 12), S. 270. 
Zufall im Namen von Rationalität, Gerechtigkeit, vulgo: Gemeinwohl völlig systemfremd wäre.

Die Koinzidenz von Repräsentation und Gewaltenteilung und die daraus resultierende multiple Repräsentation und gewaltenteilige Verbindung repräsentativer Ämter machen somit einen zufallsbestimmten „tie breaker“ überflüssig. Langwierige Entscheidungen, kontroverse Entscheidungen, ja, auch Entscheidungsblockaden gehören zur systemischen institutionellen Logik und zur gewollten, institutionalisierten Konkurrenz und Koordination differenter Handlungslogiken. Zu dieser sich auf Amt und Mandat erstreckenden Logik gehören die Zumutung der Entscheidung, die Zumutung schlechter Entscheidungen oder auch die Zumutung, keine Entscheidung zustande zu bekommen - all dies ist aber zurechenbar und zu verantworten. Eine ausgeloste Entscheidung ist dies nicht, ebenso wenig ein zugelostes Amt. Hier würden Amt, Entscheidung und Verantwortung entkoppelt und auf zumindest fragwürdige Weise durch den Zufall im Dienste des vermeintlichen Gemeinwohls substituiert.

\section{Von der Unnötigkeit einer zufallsgenerierten pouvoir neutre im modernen Parlamentarismus}

Und deswegen überzeugt mich auch das Argument der selektiven Entscheidungsentlastung durch Losverfahren nicht. Dies ist mit der oben erläuterten institutionellen Logik von Amt und Mandat in der repräsentativ-gewaltenteilenden Demokratie in letzter Konsequenz ebenfalls nicht vereinbar. Gerade wenn es in der Staat-Bürger-Beziehung um „hard cases“, knappe Güter oder Ressourcen geht, dürfen Entscheidungen in der repräsentativen Demokratie nicht an den Zufall oder eine durch den Zufall generierte Entscheidungsinstanz externalisiert werden - die gewählten (!) Entscheider haben ein Amt anvertraut bekommen, das von ihnen Entscheidungen fordert, die sie zu verantworten haben. Sie sollen sich darüber den Kopf zerbrechen, sich zanken und streiten und dann Entscheidungen treffen, die ihnen mit all ihren Folgen zugerechnet werden - ebenso wie das Nichtzustandekommen von Entscheidungen. Der Anspruch indessen, die repräsentative Demokratie müsse rationalere und gerechtere Entscheidungen im Sinne eines zwar nicht authentischen, aber informierten politischen Willens generieren, bürdet dem Parlamentarismus einmal mehr eine Repräsentationsaufgabe auf, die er organisatorisch nicht erbringen kann und soll.

Diese Aufgabe dann an den Zufall zu externalisieren, der als pouvoir neutre fungiere, mutet schon einigermaßen gewagt an, zumal wenn man erinnert, dass die von Buchstein beigezogene pouvoir neutre sowohl in der Variante von Benjamin Constant als auch der von Carl Schmitt gar nicht neutral ist. Ich halte somit diese Konnotierung gerade im Kontext der hier diskutierten Problematik für ausgesprochen heikel. Sowohl bei Constant als auch bei Schmitt soll die pouvoir neutre im Sinne eines höheren Ganzen agieren, wenn Krisen, Blockaden und Parteiengezänk das Regierungssystem lahmlegen. Robert Redslobs Lehre vom echten und unechten Parlamentarismus ${ }^{63}$ schuf die Grundlage dafür, dass ein Teil der Weimarer Staatsrechtslehre, angeführt von Carl Schmitt, einen echten Parlamentarismus nur dort

$63 \mathrm{Vgl}$. Robert Redslob, Die parlamentarische Regierung in ihrer wahren und in ihrer unechten Form. Eine vergleichende Studie über die Verfassungen von England, Belgien, Ungarn, Schweden und Frankreich, Tübingen 1918. 
gewährleistet sah, wo Legislative und Exekutive (legitimatorisch) gleichen Ursprungs seien und gleiches Gewicht hätten. Ein Reichspräsident als „Hüter der Verfassung “64 und mindestens ebenbürtiger Repräsentant des Volkswillens und eines „echten“ Parlamentarismus konnte seine pouvoir neutre dann aber auch flugs für eine „Kampfregierung“ gegen das Parlament einsetzen - denn er repräsentierte ja etwas, was das Parlament mit seinen Neutralitätsdefiziten und das Volk mit seinen Willensdefiziten gar nicht parat haben konnten: ein höheres Ganzes. Es dürfte Buchstein fern liegen, in diese Richtung gehen zu wollen. Das ändert aber an zweierlei nichts: Der Rückgriff auf die pouvoir neutre ist zumindest unglücklich, und eine Art höheres Ganzes ist in dem von ihm vorgeschlagenen House of Lots ebenfalls präsent. Es figuriert hier nur nicht mehr als existenzielle Einheit und authentischer Volkswille, die einen Hüter und wahren Repräsentanten brauchen, sondern als deliberativ generierte Rationalität, Gerechtigkeit oder Gleichheit, deren besserer Diener in bestimmten Situationen (Willens- und Neutralitätsdefizit) der Zufall sei. Die Basiswährung der repräsentativen Demokratie ist aber weder Rationalität noch Gerechtigkeit noch Gleichheit, sondern Vertrauen in ein vom Bürger legitimiertes und mit Herrschaftsmacht ausgestattetes Amt. ${ }^{65}$ Dieses Vertrauen, das gewährt oder entzogen werden kann, kann und sollte man nicht dem Zufall überlassen, denn das entkoppelt nicht nur Amt, Entscheidung und Verantwortung, sondern entmündigt letztlich auch den Bürger. ${ }^{66}$ Insofern antworte ich auf die Alternative „Lostrommel oder Wahlurne“ in der repräsentativen parlamentarischen Demokratie: Gehen Sie nicht über Los und überlassen Sie die Aleatorik der Lotto-Fee!

64 Carl Schmitt, Der Hüter der Verfassung, Berlin 1998, S. 132 ff.

65 Vgl. Martin Morlok, Demokratie und Wahlen, in: Peter Badura / Horst Dreier (Hrsg.), Festschrift 50 Jahre Bundesverfassungsgericht, Bd. 2: Klärung und Fortbildung des Verfassungsrechts, Tübingen 2001, S. $559-608$, S. $572 \mathrm{ff}$.

66 Eine Konsequenz, die aus neo-republikanischer Sicht und ebenso aus Sicht der deliberativen Demokratie ausgesprochen schmerzhaft sein dürfte. 